\title{
p63 in epithelial development
}

Eleonora Candi ${ }^{1,4}$, Rita Cipollone ${ }^{1}$, Pia Rivetti di Val Cervo ${ }^{1}$, Stefania Gonfloni², Gerry Melino ${ }^{1,3}$ and Richard Knight ${ }^{3}$

1 Biochemistry Laboratory IDI-IRCCS and University of Rome "Tor Vergata", 00133 Rome, Italy.

2 Department of Biology, University of Rome "Tor Vergata", 00133 Rome, Italy.

3 Medical Research Council, Toxicology Unit, Leicester University, Leicester LE1 9HN, United Kingdom.

4Corresponding Author: Dr Eleonora Candi, University of Rome "Tor Vergata", via Montpellier, 1, 00133,

Rome, Italy. Phone:+39-06-7259-6487, Fax: +39-06-2042-7290,

email: candi@uniroma2.it.

Key words: p63, epidermis, cornification, skin, development, Itch, Ubiquitin E3 ligase List of abbreviations: K, keratin; p63-/-, mouse knockout for p63; p63-/-;TA, p63 knockout mice complemented with TAp63; p63-/-; $\Delta \mathrm{N}$, p63 knockout mice complemented with $\Delta$ Np63; p63-/-;TA; $\Delta \mathrm{N}$, p63 knockout mice complemented with both TAp63 and $\Delta \mathrm{Np63;IKK \alpha}$, IkB kinase- $\alpha$; TA, transactivation domain; $\Delta \mathrm{N}$, amino-terminal truncated protein.

\begin{abstract}
The epidermis, the outer layer of the skin composed of keratinocytes, is a stratified epithelium that functions as a barrier to protect the organism from dehydration and external insults. The epidermis develops following the action of the transcription factor p63, a member of the p53 family of transcription factors. The Trp63 gene contains two promoters, driving the production of distinct proteins, one with an $\mathrm{N}$-terminal transactivation domain (TAp63) and one without (DeltaNp63), although their relative contribution to epidermal development is not clearly established. Trp63 mutations are involved in the pathogenesis of several human diseases, phenotypically characterized by ectodermal dysplasia. In this review we summarise the current advances that have been made in understanding the role of p63 in epidermal morphogenesis.
\end{abstract}




\section{Introduction}

The epidermis is a multilayered, stratified epithelium that provides a physical barrier for the organism, protecting it from dehydration and a variety of environmental insults. The epidermis is continuously regenerated by mitotically active keratinocytes that reside in the inner basal layer and which, following detachment from the basement membrane, migrate to the outer cornified layer (terminal differentiated compartment): this process is called cornification [1]. The epidermis is continuously renewed due to a regulated balance between proliferation and differentiation. The renewal capacity is due to the presence of epidermal stem cells and transient amplifying (TA) cells in the basal layer of the interfollicular epidermis and in the bulge (at least in mouse) of the hair follicle [2]. The concept that the transcription factor p63 is essential for the formation of the epidermis and other stratified epithelia arises from two lines of evidence. First, mice lacking all p63 isoforms have no epidermis [3-5] or squamous epithelia (prostate, urothelium) and also lack epithelial appendages, such as mammary, salivary and lachrymal glands, hair follicles and teeth. $\mathrm{p} 63^{-\%}$ mice also show truncated limbs and abnormal craniofacial development, due to failure to maintain or differentiate the apical ectodermal ridge, a structure which is important for coordination of epithelialmesenchymal interactions $[4,5]$ and is required for limb outgrowth and palatal and facial structure formation. Second, mutations in the human p63 gene are responsible for ectodermal dysplastic syndromes [6,7]. But how does p63 contribute to the formation of the epidermis? Through which underlying mechanism? And what is the molecular pathogenesis of ectodermal dysplasic syndromes?

In this review, we will focus on the involvement of p63 isoforms in the development of the epidermis. We will also discuss how the stability of this transcription factor is regulated in keratinocytes.

\section{Trp63 gene organization and p63 protein structure}

The human p63 gene is located on chromosome 3q27-29 and comprises 15 exons. Like the other members of the p53 family, p63 is expressed from two different promoters, P1 and P2, that generate two classes of proteins, TAp63, which contains the $\mathrm{N}$-terminal transactivation (TA) domain, and the $\mathrm{N}$-terminal truncated $(\Delta \mathrm{Np} 63)$ isoform, which lacks this transactivation domain (Fig. 1B) [8,9]. Alternative splicing at the 3 ' end of the transcripts also contributes to the diversity of the p63 protein, generating three different C-termini: $\alpha, \beta$ and $\gamma$. Both TAp63 and $\Delta$ Np63 can be alternatively spliced at the carboxy-terminus, leading to a total of six p63 isoforms (Fig. 1C).

All six p63 isoforms contain a DNA-binding domain (DBD) and an oligomerization domain (OD). The DBD has about $60 \%$ amino acid identity to the DBD of p53, while the OD has about $37 \%$ identity to that of p53. TAp63 isoforms have an $\mathrm{N}$-terminal transactivation domain (TA) with about $22 \%$ identity to the transactivation domain of $p 53$. In addition, the $\alpha$ isoforms contain a Sterile Alpha-Motif (SAM) and a Trans Inhibitory Domain (TID). The former is a protein-protein interaction domain, also present in other molecules [10,11], while the latter is an inhibitory domain that blocks transactivation by masking a few residues on the $\mathrm{N}$-terminal TA, (it could also be responsible for oligomerisation between different p63 isoforms) [12].

It is not yet possible to clearly attribute specific functions to the distinct isoforms. Although RNA transcripts of both TAp63 and $\triangle \mathrm{Np63}$ have been detected in mouse embryos and in post-developmental epithelial cells and tissues [13,14], at the protein level $\Delta \mathrm{Np63}$ is much more strongly represented than TAp63, and it remains debatable whether TAp63 is expressed at all. TAp63 is, however, strongly expressed in oocytes $[15,16]$. 
Expression of p63 isoforms during epidermal development and in adult epidermis

Epidermal development proceeds in multiple stages from a single-layered epithelium on the surface of the ectoderm which expresses keratin (K) 18 [17]. At embryonic day E9.5, stratification occurs with the formation of the periderm around the upper limb buds and the expression of the stratification markers, K5 and K14. At this stage, the developing outer layer of the embryo exhibits barrier function [18]. Subsequently, at embryonic day E15.5, the epidermis forms the spinous and granular layers with the concomitant expression of the differentiation markers, K1 and loricrin [19]. At E19.5, epidermal stratification and maturation is complete, although periderm is no longer present at this stage. In the adult, the epidermis continuously regenerates with a finely regulated differentiation program taking place progressively as committed cells in the basal compartment move upwards to the epidermal surface (Fig. 1A).

p63 isoform expression during epidermal development has been detected by RT-PCR and in situ hybridization techniques. p63 is first expressed before stratification occurs at embryonic day E8.5. RT-PCR analysis using specific probes to discriminate between TAp63 and $\Delta$ Np63 isoforms demonstrates that the latter is the main isoform expressed at all embryonic stages during epidermal, tooth and hair development, accounting for $100 \%$ of all p63 isoforms expressed up to embryonic day E9 and for $99 \%$ at embryonic day E13. TAp63 isoform expression starts at E13, and only accounts for $1 \%$ of total p63 mRNA expressed at this embryonic stage. However, TAp63 isoforms have not been detected by in situ hybridization or immunoblot; thus TAp63 protein levels appear to be barey detectable $[13,20]$. Studies in ES-derived cells shows that BMP-4 treatment, leading to the ectodermal fate, induces $\Delta \mathrm{Np} 63$, while exogenous expression of the $\Delta \mathrm{Np63}$ isoform in ES-derived ectodermal cells efficiently leads to their differentiation into keratinocytes [21].

Studies performed in the mature epidermis using antibodies directed against the C-terminal region of the p63 $\alpha$ proteins and the DNA-binding domain have shown that $\Delta \mathrm{Np63} \alpha$ is the main isoform expressed in keratinocytes of the proliferative basal layer; but its expression is reduced in the suprabasal differentiated layers of mature epidermis [22-24]. Expression of the full-length TAp63 mRNA has also been described [23,24] and some studies, using not commercially available specific anti-TAp63 antibodies, have detected TAp63 proteins in adult epidermis [22,25]. The TAp63 isoform has also been shown to be constitutively expressed in female germ cells during meiotic arrest, and is essential (after post-translational modification) for DNA damage-induced oocyte death independently of p53 [15].

\section{Differential function of p63 isoforms in epidermal formation}

Despite its structural similarity to p53, the central role of p63 in epidermal development, as opposed to tumour susceptibility, became established with the publication of two independent studies on $163^{-/}$mouse models generated by disrupting exons common to both TA and $\Delta \mathrm{N}$ isoforms [4,5]. Although the phenotype of the mice was very similar, different conclusions were reached concerning the molecular mechanism.

Both groups of mice were born alive but died within hours of birth because they failed to develop skin. However, in the $p 63^{-/-}$mice developed by McKeon's lab [4] some patches of the skin expressed markers of differentiation including loricrin, involucrin, and filaggrin. Thus, it was concluded that the lack of p63 does not affect the differentiation process but impairs the proliferation potential of stem cells. In contrast, Roop's lab [5] did not detect any markers of skin differentiation such as loricrin or involucrin, and suggested that, in the absence of p63, skin development aborts at an early stage without starting a pluristratification program, and that p63 is therefore required for the commitment of the immature ectoderm to the epidermal lineage. 
Furthermore, one group claimed that TAp63 isoforms appeared first during embryogenesis and were required for epithelial stratification [13,22]. Recently, McKeon's lab demonstrated [26], that p63 was strongly expressed in epithelial cells with high clonogenic and proliferative capacity and that $\mathrm{p} 63^{--}$epithelial stem cells undergo premature proliferation rundown, both is epidermis and in thymus, indicating that p63 is a key determinant of the proliferative capacity in epithelia stem cells [26]. Indeed, a detailed analysis of the expression of $\mathrm{K} 5$ and loricrin in the epidermis of $\mathrm{E} 16 \mathrm{wt}$ and $\mathrm{p} 63^{-/-}$mice, revealed that cells remaining on the surface of the p63 embryos are committed to an epidermal lineage that progress through differentiation [26].

We have obtained similar evidences using a different approach, we complemented p63deficient mice by expressing TAp63 and $\Delta$ Np63 transgenes under the control of the K5 promoter [27]. Hence, by crossing TAp63 $\alpha$ or $\Delta \mathrm{Np} 63 \alpha$ epidermal transgenic mice into p63-/- mice, we generated mice expressing one single isoform to identify the relative contribution of each isoform to the development of the epidermis and in thymic epithelial cells $[27 ; 28]$. Although we were unable to obtain a full reversion of the phenotype of the null mice, the study showed that transgenic mice complemented with $\Delta \mathrm{Np} 63\left(\mathrm{p} 63^{-/-} ; \Delta \mathrm{N}\right)$ developed a significant basal layer, while transgenic mice complemented with TAp63 $\left(\mathrm{p} 63^{-/} ; \mathrm{TA}\right)$ did not differ from $\mathrm{p} 63^{-/-}$mice. Double complemented mice exhibited a greater degree of re-epithelialization with expression of keratins of the basal layer (K5 and K14), and differentiation-specific markers such as $\mathrm{K} 1$ and loricrin but complete cornification remained impaired [27]. These data suggest that, although $\Delta \mathrm{Np} 63 \alpha$ plays a pivotal role in development, any contribution by TAp63 $\alpha$ is only concomitant or subsequent to the function of $\Delta \mathrm{Np63 \alpha}$. We therefore asked which genes are transcriptional targets of p63. In addition to TAp63, $\Delta \mathrm{Np} 63$ also showed transcriptional activity which is due to the presence of a C-terminal TA domain see Fig.1C. Analysis of target genes induced by either TAp63 or $\triangle \mathrm{Np63}$ in Saos2 cells showed that TAp63a mainly regulates terminal differentiation markers such as $\mathrm{K} 1, \mathrm{~K} 10$, profilaggrin and involucrin, while $\Delta \mathrm{Np} 63 \alpha$ induces the early differentiation marker $\mathrm{K} 14$ [27]. These data suggested that $\Delta \mathrm{Np63}$ is important for maintaining the proliferative potential of the basal layer, whereas TAp63 contributes by acting synergistically and/or subsequently to $\Delta$ Np63 to allow differentiation [27]. Furthermore, these results are consistent with a study based on RNA interference directed against p63 isoforms, in which the authors demonstrated that $\Delta$ Np63 isoforms are the main mediators of p63 effects and that TAp63 isoforms contribute to later stages of differentiation in mature keratinocytes. In the same work the roles of p63 in differentiation and cell proliferation were shown to be separated, indeed down-regulation of p63 causes a cell cycle arrest in keratinocytes that is p53-dependent [29;30]. Simultaneous knockdown of p53 in the context of p63 loss is able to rescue cell proliferation, however the differentiation defect is still present [29]. These data [27,29] are therefore somewhat in contrast with the results from Roop's group [24,31], who showed that, in vivo siRNA for TAp63 produced a dramatic phenotype, with failure to organize pluristratified epithelia but no significant effect was observed on the basal layer. However, in our model [27] we investigated the role in early development using a genetically pure model, with the total absence of one isoform, the elegant in vivo study by Roop using siRNA addressed a slightly different question, representing a model of isoform unbalance.

\section{Transcriptional regulation by p63}

\section{Transcriptional activities of p63 isoforms}

For long time, $\Delta \mathrm{Np63}$ was thought unable to drive the expression of target genes due to the lack of a transactivation domain. The accepted activity was its dominant negative effect exerted towards the TAp63 isoform, as suggested by co-transfection experiments in luciferase reporter assays [3]. However, in addition to the competition 
between TAp63 and $\triangle \mathrm{Np63}$ isoforms, growing evidence indicated that these $\mathrm{N}$-terminal isoforms had different transcriptional specificities a specificity confirmed by functional domain characterization [32-34], and microarray analysis showing that both isoforms can induce gene transcription [35,29]. As expected from genes important for developmental and differentiation processes, the more strongly represented classes of target genes are those concerned with cell adhesion, the cytoskeleton, and the cell cycle. Moreover, as p63 is a master gene regulator, it is not surprising that many of its targets are themselves transcription factors or co-regulators.

Depending on the specific p63 isoform and promoter being tested, p63 can either be a transcriptional activator or repressor. Additionally, emerging evidence underlines the importance of $\Delta N p 63$ interaction with other transcription factors by which its functional activity can be modulated. For instance, in the case of HSP70 transcriptional regulation, $\Delta$ Np63 interacts with the NF-Y transcription factor to form a complex with the CCAAT box located in the HSP70 promoter, thus enhancing HSP70 expression [36,37]. In contrast, the same functional interaction between $\triangle \mathrm{Np} 63 \alpha$ and the CCAAT binding factor NF-Y, again at the level of CCAAT boxes, determines the repression of transcription of cell cycle-related genes such as cyclin B2, Cdc2 and topoisomerase II [37].

\section{p63 target genes relevant in epidermal formation and differentiation}

While it is indisputable that the p63 gene plays a master role in epidermal development, the molecular mechanisms underlying the regulation of the development and maintenance of the epidermis are still under investigation. In this context, a genomewide approach led to the identification of several high-confidence target genes for $\Delta N p 63$ [35]. However, only a few of these have been demonstrated to have a defined impact on epidermal formation and differentiation in vivo.

In proliferating primary keratinocytes $\Delta \mathrm{Np} 63 \alpha$ interacts with and represses the promoters of $\mathrm{p} 21^{\mathrm{WAF} 1 / \mathrm{Cip} 1}$ and $14-3-3 \sigma$, two genes required for keratinocyte terminal differentiation. Thus, a reduced proliferative capacity of $\mathrm{p} 63^{-/-}$mouse keratinocytes could be the consequence of an increased expression of p21 [38]. In fact, it has been shown that the blockade of cell proliferation due to lack of p63, is p53-dependent. Consistently, p53 down regulation rescues the cell proliferation defect due to a p63 knockdown, but not the incapacity of p63-less cells to differentiate [29, 30]. An additional signaling pathway playing a crucial role in epidermal stratification and keratinocytes differentiation is through Notch [39-43]. Although several evidences indicate that $p 63$ functions as a modulator of Notch1-dependent transcription and function and also directly transactivates the Notch ligand, JAGG-1 [39], the role of p63 in modulating Notch signaling is not clear. It has been shown that p63 functions as selective modulator of Notch-dependent transcription and function through the negative regulation of Hes-1 by p63 [40] in keratinocytes. However, in vivo studies demonstrated that absence of Notch pathway did not lead to a decrease of p63 expression [43].

$\mathrm{IKK} \alpha\left(\mathrm{I}_{K} \mathrm{~B}\right.$ kinase- $\left.\alpha\right)$ is also a p63 target gene. Like p63, IKK $\alpha$ is essential for normal epidermal development [44]. The phenotypic similarities between $\mathrm{p} 3^{-/-}$and IKK $\alpha^{-}$ /- mice suggested that IKK $\alpha$ may be a downstream target of p63 that is positively regulated by both TAp63 and $\triangle \mathrm{Np63}$. p63 regulation of IKK $\alpha$ was both direct and indirect, as well as isoform-specific (Fig. 2). Indeed, TAp63 directly transactivates IKK $\alpha$ by binding a p53-like consensus sequence on the IKK $\alpha$ promoter. In addition TAp63 also transactivates IKK $\alpha$ indirectly through the induction of Ets-1 and GATA-3, whose consensus binding sites are present in the IKK $\alpha$ promoter [45,46] (Fig. 2). GATA-3 is an important mediator of skin and hair development [47], although its specific function in epidermal development and differentiation has not yet been established. However, $\Delta$ Np63 can transactivate IKK $\alpha$ only indirectly via GATA-3 (Fig. 2). 
The p53 responsive transmembrane protein Perp is also positively regulated by both TAp63 and $\Delta$ Np63, and Perp $^{-/-}$mice exhibit blistered skin, a stratified epithelium characteristic of compromised adhesion [48]. Other epithelial genes regulated by p63 are: $\mathrm{K} 14$, a marker of the basal layer [27], envoplakin, an early envelope precursor [49], and BPAG-1, a component of hemidesmosomes [50].

\section{p63 protein degradation}

\section{Regulation of epidermal keratinocyte differentiation}

Given the self-renewal properties of the epidermis, p63 expression must be finely tuned. We have identified Itch as the ubiquitin E3 ligase responsible for the degradation of p63 [51]. Itch, a HECT type E3 ligase, also known as atrophin-1 interacting protein 4 (AIP4), typically regulates the stability of both transmembrane receptors through monoor multi-ubiquitylation, and intracellular substrates via mono- or poly-ubiquitylation, driving them to proteasomal degradation. Itch possesses a WW domain which preferentially binds a PPXY consensus sequence in its substrates. p63 displays a unique PY motif spanning residues 501-504 (TAp63 sequence) which render the protein susceptible to Itch-mediated ubiquitylation and degradation [51] (Fig. 3A). p63 and Itch distribution in the adult human normal epidermis is respectively predominant in the basal and upper layers [51] (Fig. 3B). Intriguingly, a number of Itch substrates (c-Jun, JunB, Notch, Gli1) are transcription factors controlling epidermal stem cell maintenance and keratinocyte specification, as well as orchestrating the spatio-temporal progression of terminal differentiation [52]. It is therefore likely that Itch-mediated degradation would have a regulatory role in skin biology $[40,43,51,53]$. Moreover, the in vivo expression gradient of the substrate (p63) and the ligase (Itch) parallels their expression pattern during keratinocyte differentiation, when an accumulation of Itch is accompanied by a reduction of $\Delta \mathrm{Np} 63$ and by the expression of keratin 1 and involucrin (Fig. 3C-D). Recently we have also identified a binding patner of Itch able to inhibit its function: N4BP1 [54]. While there is good evidence on how N4BP1 regulates Itch function, there is currently no evidence on its role in the epidemis. Altogether, these observations strongly indicate that Itch physiologically controls p63 steady-state protein levels, and therefore, the capacity of the transcription factor to direct the expansion of the basal compartment [51]. In addition it has been very recently published that p63 is not only regulated by post-translational mechanisms, but also post-transcriptionally through the microRNA machinery. miR 203 targets the 3'UTR of the p63 mRNA and most likely blocks its translation process [52]. The role of miR-203 was also studied in vivo by ectopic expression under the K14 promoter and it resulted in a thinner skin with aberrant expression of differentiation markers throughout the epidermal layers, and most transgenic mice died shortly after birth. The depletion of basal stem cells in transgenic mice reminds of the p63-/phenotype, and indeed, these mice show low levels of p63 in basal cells [53].

\section{Conclusion}

From an evolutionary perspective, p63 not only belongs to the p53 family but it is the founding member of the family, from which p53 evolved to specifically act in DNA damage by regulating cell cycle arrest and cell death. p63, the more ancestral member of the family, is a key regulator of the expansion of the basal keratinocyte population and is essential for the development of several different epithelia. In the skin, p63 functions as a key regulatory element of cellular proliferation, differentiation and survival in both physiological and pathological contexts. Despite accumulating evidence about the p63 network, the molecular mechanisms by which p63 participates in epidermal self-renewal and homeostasis are complex and still far from being fully understood. The discovery of Trp63 has indeed opened a new theme of research. 


\section{Acknowledgements}

We thank Dr Alessandro Giamboi-Miraglia for technical support. The work discussed in this manuscript has been supported by the Medical Research Council (G.M.) and by grants from Telethon (GGP02251 to EC \& GGPO4110 to GM), AIRC (2743), EU (LSGBH-2005-019067-Epistem; LSHC-CT-2004-503576-Active p53), MIUR, PRIN 06 to EC, PRIN 06 to GM and MinSan to G.M.

\section{References}

[1] Candi, E., Schmidt, R. and Melino, G. (2005) The cornified envelope: a model of cell death in the skin. Nat. Rev. Mol. Cell Biol. 6, 328-340.

[2] Blanpain, C. and, Fuchs E. (2006) Epidermal stem cells of the skin. Annu. Rev. Cell Dev. Biol. 22, 339-373.

[3] Yang, A., Kaghad, M., Wang, Y., Gillett, E., Fleming, M. D., Dötsch, V., Andrews, N. C., Caput, D. and McKeon, F. (1998) p63, a p53 homolog at 3q27-29, encodes multiple products with transactivating, death-inducing, and dominant-negative activities. Mol. Cell 2, 305-316.

[4] Yang, A., Schweitzer, R., Sun, D., Kaghad, M., Walker, N., Bronson, R. T., Tabin, C., Sharpe, A., Caput, D., Crum, C. and McKeon, F. (1999) p63 is essential for regenerative proliferation in limb, craniofacial and epithelial development. Nature 398, 714-718.

[5] Mills, A. A., Zheng, B., Wang, X. J., Vogel, H., Roop, D. R. and Bradley, A. (1999) p63 is a p53 homologue required for limb and epidermal morphogenesis. Nature 398, 708713.

[6] Celli, J., Duijf, P., Hamel, B. C., Bamshad, M., Kramer, B., Smits, A. P., NewburyEcob, R., Hennekam. R. C., Van Buggenhout, G., van Haeringen, A., Woods, C. G., van Essen, A. J., de Waal, R., Vriend, G., Haber, D. A., Yang, A., McKeon, F., Brunner, H. G. and van Bokhoven. H. (1999) Heterozygous germline mutations in the p53 homolog p63 are the cause of EEC syndrome. Cell 99, 143-153.

[7] Rinne, T., Brunner, H. G. and van Bokhoven, H. (2007) p63-associated disorders. Cell Cycle 6, 262-268.

[8] Yang, A. and McKeon, F. (2000) P63 and P73: P53 mimics, menaces and more. Nat. Rev. Mol. Cell Biol. 1, 199-207.

[9] Yang, A., Kaghad, M., Caput, D. and McKeon, F. (2002) On the shoulders of giants: p63, p73 and the rise of p53. Trends Genet. 18, 90-95.

[10] Schultz, J., Ponting, C. P., Hofmann, K. and Bork P. (1997) SAM as a protein interaction domain involved in developmental regulation. Protein Sci. 6, 249-253.

[11] Thanos, C. D. and Bowie, J. U. (1999) p53 family members p63 and p73 are SAM domain-containing proteins. Protein Sci. 8, 1708-1710.

[12] Serber Z, Lai HC, Yang A, Ou HD, Sigal MS, Kelly AE, Darimont BD, Duijf PHG, van Bokhoven H, McKeon F, Dotsch V. (2002) A C-terminal inhibitory domain controls the activity of p63 by an intramolecular mechanism Mol Cell Biol 22(24):8601-8611

[13] Laurikkala M. (2007) p63 in skin appendage development. Cell Cycle 6, 285-290. 
[14] Koster, M. I, Dai, D. and Roop, D. R. (2007) Conflicting roles for p63 in skin development and carcinogenesis: are the mouse models at fault? Cell Cycle 6, 269-273.

[15] Suh, E. K., Yang, A., Kettenbach, A., Bamberger, C., Michaelis, A. H., Zhu, Z., Elvin, J. A., Bronson, R. T., Crum, C. P. and McKeon, F. (2006) p63 protects the female germ line during meiotic arrest. Nature 444, 624-628.

[16] Livera G, Petre-Lazar B, Guerquin MJ, Trautmann E, Coffigny H, Habert R (2008) p63 null mutation protects mouse oocytes from radio-induced apoptosis. Reproduction. 135(1):3-12.

[17] Moll, R., Franke, W. W., Schiller, D. L., Geiger, B. and Krepler, R. (1982) The catalog of human cytokeratins: patterns of expression in normal epithelia, tumors and cultured cells. Cell 31, 11-24.

[18] Bickenbach, J. R., Greer, J. M., Bundman, D. S., Rothnagel, J. A., Roop, D. R., Byrne, C., Tainsky, M. and Fuchs, E. (1994) Programming gene expression in developing epidermis. Development 120, 2369-2383.

[19] Bickenbach, J. R., Greer, J. M., Bundman, D. S., Rothnagel, J. A. and Roop, D. R. (1995) Loricrin expression is coordinated with other epidermal proteins and the appearance of lipid lamellar granules in development. J. Invest. Dermatol. 104, 405-410.

[20] Laurikkala, J., Mikkola, M. L., James, M., Tummers, M., Mills, A. A. and Thesleff, I. (2006) p63 regulates multiple signalling pathways required for ectodermal organogenesis and differentiation. Development 133, 1553-1563.

[21] Aberdam D, Gambaro K, Rostagno P, Aberdam E, de la Forest Divonne S, Rouleau M. (2007) Key roe of p63 in BMP-4 induced epidermal commitment of embryonic stem cells. Cell Cycle 6: 291-294

[22] Nylander, K., Vojtesek, B., Nenutil, R., Lindgren, B., Roos, G., Zhanxiang, W., Sjostrom, B., Dahlqvist, A. and Coates, P. J. (2002) Differential expression of p63 isoforms in normal tissues and neoplastic cells. J. Pathol. 198, 417-427.

[23] Thurfjell, N., Coates, P. J., Uusitalo, T., Mahani, D., Dabelsteen, E., Dahlqvist, A., Sjostrom, B., Roos, G. and Nylander, K. (2004) Complex p63 mRNA isoform expression patterns in squamous cell carcinoma of the head and neck. Int. J. Oncol. 25, 27-35.

[24] Koster, M. I., Kim, S., Mills, A. A., DeMayo, F. J. and Roop, D. R. (2004) p63 is the molecular switch for initiation of an epithelial stratification program. Genes Dev. 18, 126.

[25] Gu, X., Lundqvist, E. N., Coates, P. J., Thurfjell, N., Wettersand, E. and Nylander, K. (2006) Dysregulation of TAp63 mRNA and protein levels in psoriasis. J. Invest. Dermatol. 126, 137-141.

[26] Senoo M, Pinto F, Crum CP, McKeon F. (2007) p63 Is essential for the proliferative potential of stem cells in stratified epithelia. Cell 4;129(3):523-36.

[27] Candi, E., Rufini, A., Terrinoni, A., Dinsdale, D., Ranalli, M., Paradisi, A., De Laurenzi, V., Spagnoli, L. G., Catani, M. V., Ramadan, S., Knight, R. A. and Melino, G. (2006) Differential roles of p63 isoforms in epidermal development: selective genetic complementation in p63 null mice. Cell Death Differ. 13, 1037-1047.

[28] Candi, E., Rufini, A., Terrinoni, A., Giamboi-Miraglia, A., Lena, A.M., Mantovani, R., Knight, R., Melino, G. (2007) DeltaNp63 regulates thymic development through 
enhanced expression of FgfR2 and Jag2. Proc Natl Acad Sci U S A. 104(29):119992004.

[29] Truong, A. B., Kretz, M., Ridky, T. W., Kimmel, R. and Khavari, P. A. (2006) p63 regulates proliferation and differentiation of developmentally mature keratinocytes. Genes Dev. 20, 3185-3197.

[30] Lee $H$, Kinelman D. (2002) A dominant negative form of $p 63$ is required for epidermal proliferation in Zebrafish. Developmental Cell 2:607-616

[31] Koster MI, Dai D, Marinari B, Sano Y, Costanzo A, Karin M, Roop DR (2007a) p63 induces key target genes required for epidermal morphogenesis. Proc Natl Acad Sci U $S$ A. 104(9):3255-60.

[32] Ghioni, P., Bolognese, F., Duijf, P. H., Van Bokhoven, H., Mantovani and R., Guerrini, L. (2002) Complex transcriptional effects of p63 isoforms: identification of novel activation and repression domains. Mol. Cell Biol. 22, 8659-8668.

[33] King, K. E., Ponnamperuma, R. M., Yamashita, T., Tokino, T., Lee, L. A., Young, M. F. and Weinberg, W. C. (2003) deltaNp63alpha functions as both a positive and a negative transcriptional regulator and blocks in vitro differentiation of murine keratinocytes. Oncogene 22, 3635-3644.

[34] Helton, E. S., Zhu, J. and Chen, X. (2006) The unique NH2-terminally deleted (DeltaN) residues, the PXXP motif, and the PPXY motif are required for the transcriptional activity of the DeltaN variant of p63. J. Biol. Chem. 281, 2533-2542.

[35] Viganò, M. A., Lamartine, J., Testoni, B., Merico, C., Alotto, D., Castagnoli, C., Robert, A., Candi, E., Melino, G., Gigrol, X. and Mantovani, R. (2006) New p63 targets in keratinocytes identified by genome-wide approach. EMBO J. 25, 5105-5116.

[36] Osada, M., Guo, Z., Fomenkov, A., Begum, S., Zhao, M., Upadhyay, S., Xing, M., Wu, F., Moon, C., Westra, W. H., Koch, W. M., Mantovani, R., Califano, J. A., Ratovitski, E., Sidransky, D. and Trink, B. (2005) DeltaNp63alpha up-regulates the Hsp70 gene in human cancer. Cancer Res. 65, 758-766.

[37] Testoni, B. and Mantovani, R. (2006) Mechanisms of transcriptional repression of cell-cycle G2/M promoters by p63. Nucleic Acids Res. 34 928-938.

[38] Westfall, M. D., Mays, D. J., Sniezek, J. C. and Pietenpol, J. A. (2003) The Delta Np63 alpha phosphoprotein binds the p21 and 14-3-3 sigma promoters in vivo and has transcriptional repressor activity that is reduced by Hay-Wells syndrome-derived mutations. Mol. Cell Biol. 23, 2264-2276.

[39] Sasaki, Y., Ishida, S., Morimoto, I., Yamashita, T., Kojima, T., Kihara, C., Tanaka, T., Imai, K., Nakamura, Y. and Tokino, T. (2002) The p53 family member genes are involved in the Notch signal pathway. J. Biol. Chem. 277, 719-724.

[40] Nguyen, B. C., Lefort, K., Mandinova, A., Antonini, D., Devgan, V., Della Gatta, G., Koster, M. I., Zhang, Z., Wang, J., Tommasi di Vignano, A., Kitajewski, J., Chiorino, G., Roop, D. R., Missero, C. and Dotto, G. P. (2006) Cross-regulation between Notch and p63 in keratinocyte commitment to differentiation. Genes Dev 20, 1028-1042. 
[41] Truong AB, Khavari PA (2007) Control of keratinocyte proliferation and differentiation by p63. Cell Cycle 6(3):295-9.

[42] Okuyama R, Ogawa E, Nagoshi H, Yabuki M, Kurihara A, Terui T, Aiba S, Obinata M, Tagami H, Ikawa S (2007) p53 homologue, p51/p63, maintains the immaturity of keratinocyte stem cells by inhibiting Notch1 activity. Oncogene 26(31):4478-88.

[43] Blanpain, C,, Lowry, W.E., Pasolli, H.A., Fuchs, E. (2006) Canonical notch signaling functions as a commitment switch in the epidermal lineage. Genes Dev. 20(21):3022-35.

[44] Sil AK, Maeda S, Sano Y, Roop DR, Karin M. (2004) IkappaB kinase-alpha acts in the epidermis to control skeletal and craniofacial morphogenesis. Nature 428:660-4.

[45] Gu, L., Zhu, N., Findley, H. W., Woods, W. G. and Zhou, M. (2004) Identification and characterization of the IKKalpha promoter: positive and negative regulation by ETS-1 and p53, respectively. J. Biol. Chem. 279, 52141-52149.

[46] Candi, E., Terrinoni, A., Rufini, A., Chikh, A., Lena, A. M., Suzuki, Y., Sayan, B. S., Knight, R. A. and Melino, G. (2006a) p63 is upstream of IKK $\alpha$ in epidermal development. J. Cell Sci. 119, 4617-4622.

[47] Kaufman, C. K., Zhou, P., Pasolli, H. A., Rendl, M., Bolotin, D., Lim, K. C., Dai, X., Alegre, M. L. and Fuchs, E. (2003) GATA-3: an unexpected regulator of cell lineage determination in skin. Genes Dev. 17, 2108-2122.

[48] Ihrie, R. A., Marques, M. R., Nguyen, B. T., Horner, J. S., Papazoglu, C., Bronson, R. T., Mills, A. A., Attardi, L. D. (2005) Perp is a p63-regulated gene essential for epithelial integrity. Cell 120, 843-856.

[49] Carroll, D. K., Carroll, J. S., Leong, C. O., Cheng, F., Brown, M., Mills, A. A., Brugge, J. S. and Ellisen, L. W. (2006) p63 regulates an adhesion programme and cell survival in epithelial cells. Nat. Cell Biol. 8, 551-561.

[50] Osada, M., Nagakawa, Y., Park, H. L., Yamashita, K., Wu, G., Kim, M. S., Fomenkov, A., Trink, B. and Sidransky, D. (2005) p63-specific activation of the BPAG-1e promoter. J. Invest. Dermatol. 125, 52-60.

[51] Rossi, M., Aqeilan, R. I., Neale, M., Candi, E., Salomoni, P., Knight, R. A., Croce, C. M. and Melino, G. (2006) The E3 ubiquitin ligase Itch controls the protein stability of p63. Proc. Natl. Acad. Sci. U S A. 103, 12753-12758.

[52] Qiu, L., Joazeiro, C., Fang, N., Wang, H. Y., Elly, C., Altman, Y., Fang, D., Hunter, T. and Liu, Y. C. (2000) Recognition and ubiquitination of Notch by Itch, a hect-type E3 ubiquitin ligase. J. Biol. Chem. 275, 35734-35737.

[53] Okuyama, R., Tagami, H. and Aiba, S. (2007) Notch signaling: Its role in epidermal homeostasis and in the pathogenesis of skin diseases. J. Dermatol. Sci. Jul 9; [Epub ahead of print].

[54] Oberst A, Malatesta $M$, Aqeilan RI, Rossi $M$, Salomoni $P$, Murillas $R$, Sharma $P$, Kuehn MR, Oren M, Croce CM, Bernassola F, Melino G. (2007) The Nedd4-binding partner 1 (N4BP1) protein is an inhibitor of the E3 ligase Itch. Proc Natl Acad Sci U S A. 104(27):11280-5. 
[52] Lena, A.M., Shalom-Feuerstein, R., Rival Cervo, P., Aberdam, D., Knight, R.A., Melino, G., Candi, E. (2008) miR-203 represses "stemness" by repressing $\Delta$ Np63. Cell Death Differ.in press

[53] Yi, R., Poy, M.N., Stoffel, M., Fuchs, E. (2008) A skin microRNA promotes differentiation by repressing 'stemness'. Nature. 452(7184):225-9.

\section{FIGURE LEGENDS}

\section{Figure 1}

(A) The epidermis is formed by layers of proliferating and differentiating keratinocytes, which have to be constantly renewed by the action of epidermal stem cells that are located in the basal layer of the epidermis. Stem cells are not the only epidermal cells that are competent to proliferate: the progeny of a stem cell that is destined to terminally differentiate may first undergo a few rounds of division, during which time they are known as transit amplifying (TA) cells. bm= basal membrane; bl= basal layer of keratinocytes; $\mathrm{CE}=$ cornified envelope; TA cells $=$ transient amplifying cells.

(B) Structure of p63 gene. The alternative promoters P1 and P2 drive the expression of transactivating (TA) and the $\mathrm{N}$-terminally truncated $(\Delta \mathrm{N})$ isoforms. Variants $(\alpha, \beta$, and $\gamma)$ arise from the alternative splicing at the 3 ' end of the gene.

(C) Schematic representation of the modular structure of the TAp63a and $\Delta N p 63 \alpha$ proteins. The main structural domains are indicated: transactivation domain (TA), DNAbinding domain, oligomerization domain (OD), second transactivation domain (TA2), sterile a motif (SAM), and transinhibitor domain (TID). The additional SAM and the TI domain are exclusive to the $\alpha$ isoforms.

\section{Figure 2}

The epidermis is maintained throughout adult life by stem cells, which self-renew and produce progeny that undergoes terminal differentiation. A simplified scheme of the actions of p63 in epidermal development, with particular emphasis on IKK $\alpha$ and $\mathrm{K} 14$, is shown here. Several studies have been performed to identify the target promoters of p63. Here is shown a simplified and limited list of published p63 target genes which have been experimentally validated. The scheme does not necessarily imply that other target genes may be less important.

\section{Figure 3}

Itch degrades p63. (A) Schematic representation of the modular structure of the TAp63a and $\Delta$ Np63a proteins. The main structural domains are indicated as in figure 2 . The p63 region containing the PPXY motif is also shown (in the box). (B) Human adult skin sections stained with monoclonal mouse anti-Itch antibodies, secondary goat anti-mouse Alexa Fluor 488, rabbit polyclonal anti-p63 (H129), and secondary goat anti-rabbit Alexa Fluor 568. Nuclei were counterstained with DAPI and analyzed by confocal microscopy. Human primary keratinocyte extracts were induced to differentiate for 3 and 5 days in a high calcium concentration and Western blotting was performed with antibodies against Itch, p63, involucrin and $\alpha$-tubulin (loading control). (D) The scheme indicates the existence of an opposing gradient between Itch and p63 in the epidermis. 
A

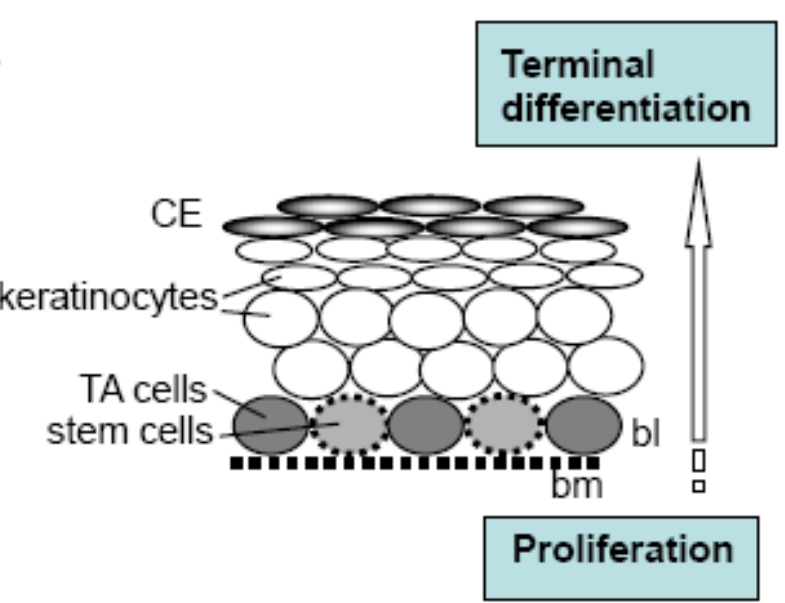

B

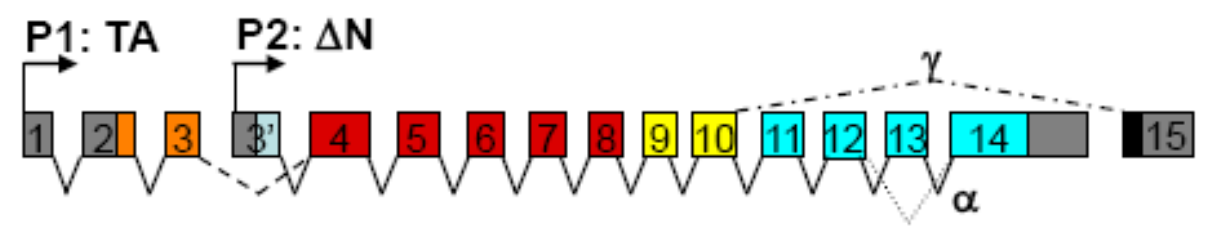

C

$\beta$

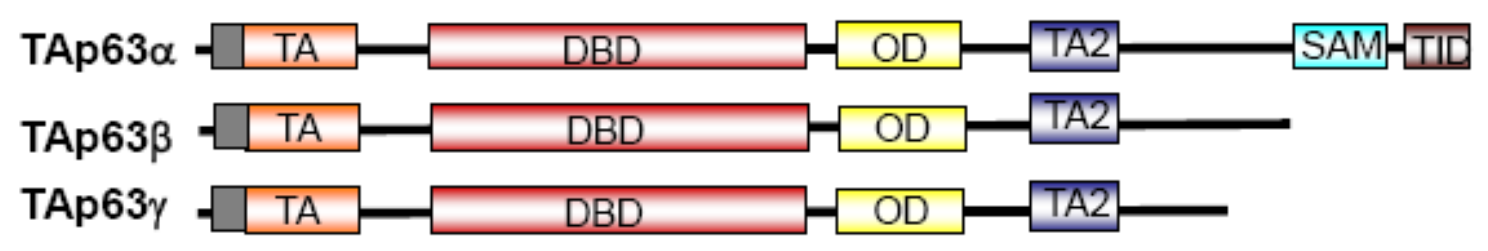

$\Delta \mathrm{Np63 \alpha}$

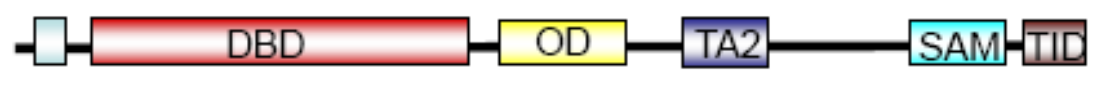

$\Delta \mathrm{Np63 \beta}$

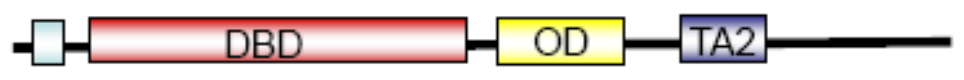

$\Delta \mathrm{Np63 \gamma}$

$\square-\square \quad \mathrm{DBD}-\mathrm{OD}-\mathrm{TA2}$

Figure 1 


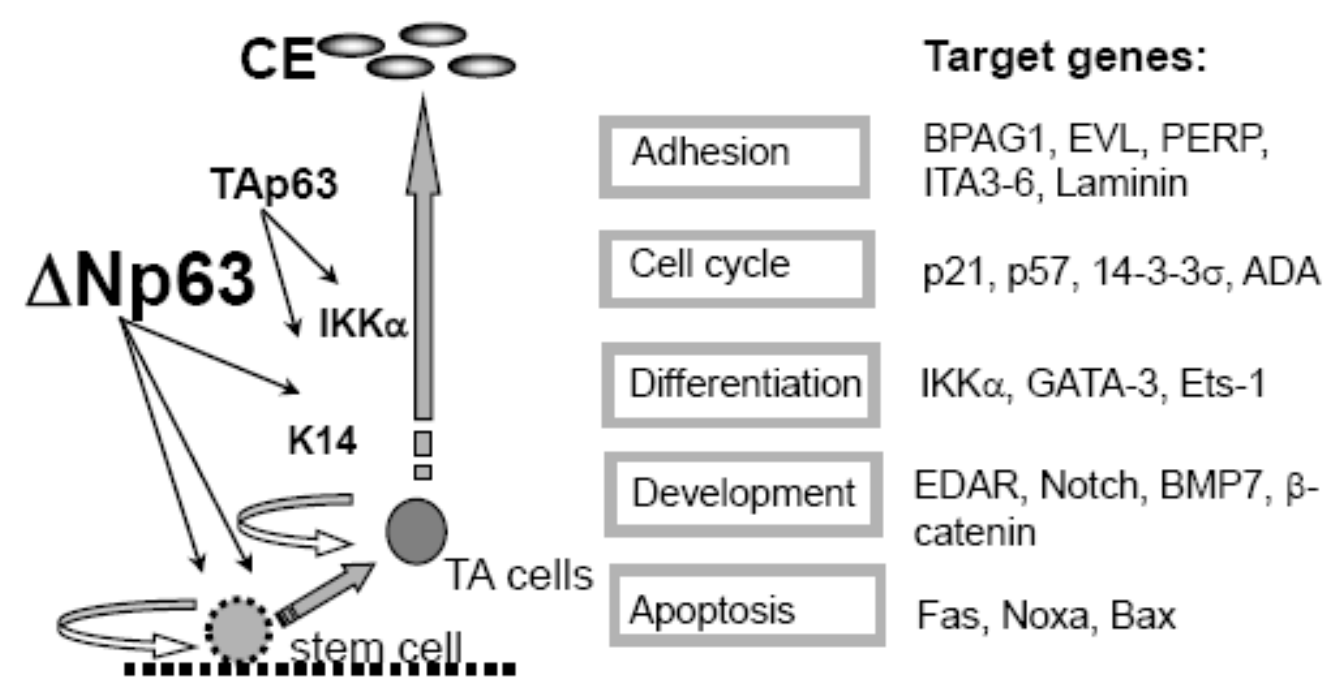

Figure 2 


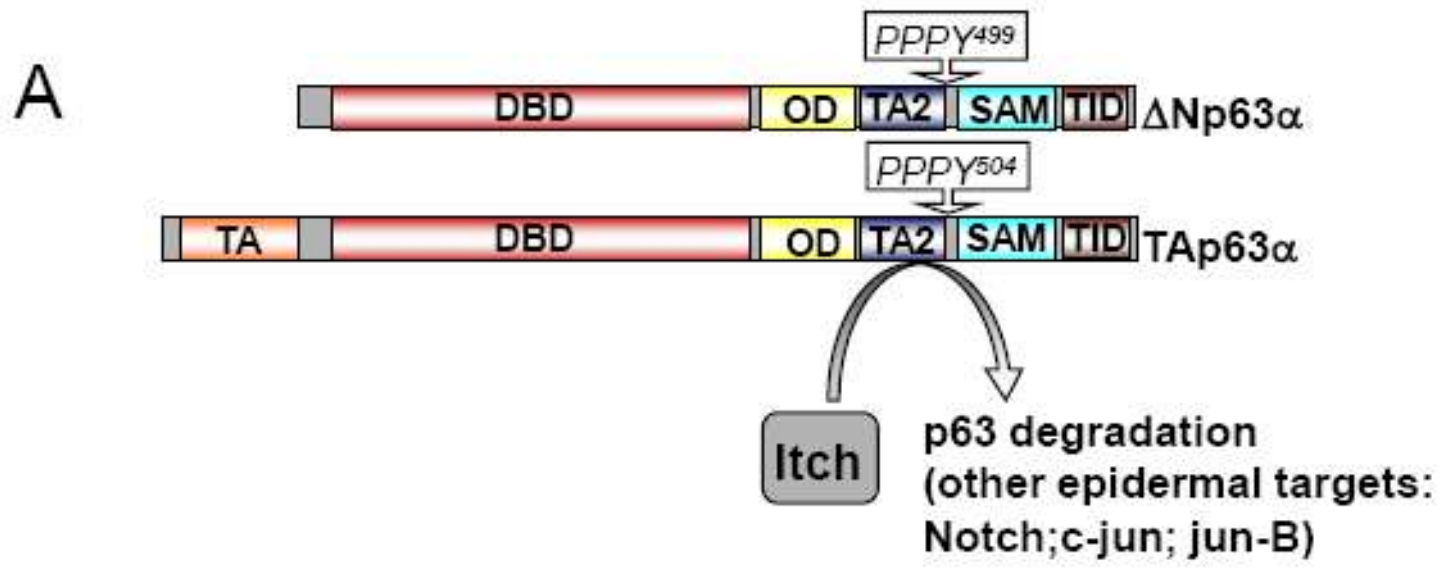

B
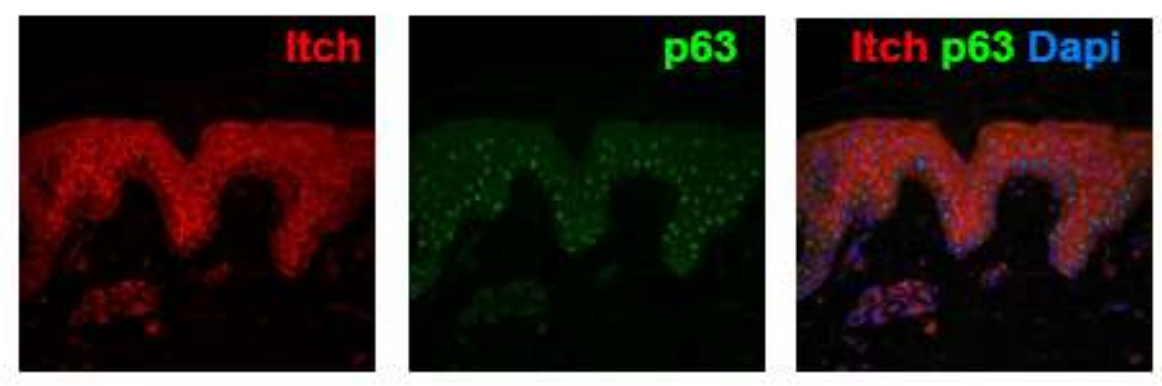

C Days of differentiation (human keratinocytes):

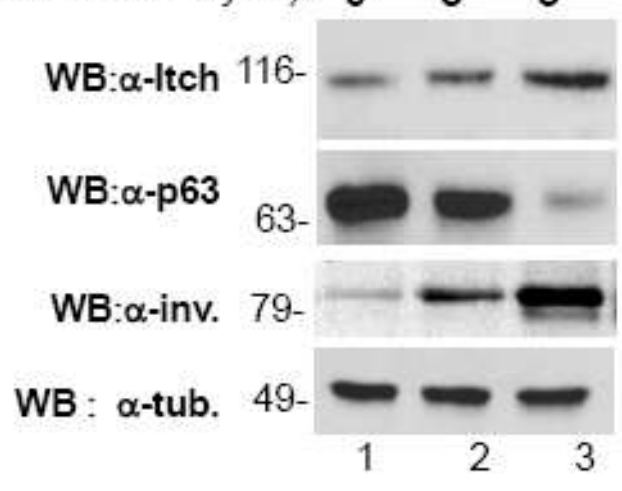

D

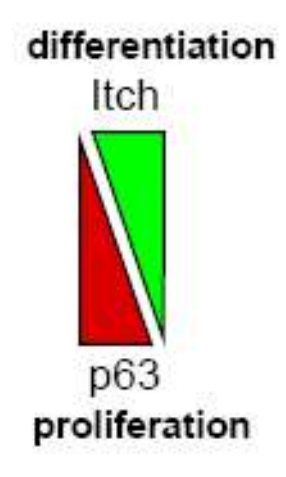

Figure 3 